Journal for ImmunoTherapy of Cancer

\title{
In silico modeling of combination systemic therapy for advanced renal cell carcinoma
}

\author{
Ritesh R Kotecha (D) , ${ }^{1}$ Dennis J Hsu (D) , , ${ }^{1,2}$ Chung-Han Lee, ${ }^{1}$ Sujata Patil, ${ }^{3}$ \\ Martin H Voss (10) ${ }^{1}$
}

To cite: Kotecha RR, Hsu DJ, Lee C-H, et al. In silico modeling of combination systemic therapy for advanced renal cell carcinoma. Journal for ImmunoTherapy of Cancer 2021;9:e004059. doi:10.1136/ jitc-2021-004059

- Additional supplemental material is published online only. To view, please visit the journal online (http://dx.doi.org/10. 1136/jitc-2021-004059).

RRK and DJH are joint first authors.

Accepted 20 November 2021

Check for updates

(c) Author(s) (or their employer(s)) 2021. Re-use permitted under CC BY-NC. No commercial re-use. See rights and permissions. Published by BMJ.

${ }^{1}$ Department of Medicine, Memorial Sloan Kettering Cancer Center, New York, New York, USA

${ }^{2}$ School of Medicine, University of Pittsburgh, Pittsburgh, PA, USA

${ }^{3}$ Department of Epidemiology and Biostatistics, Memorial Sloan Kettering Cancer Center, New York, New York, USA

Correspondence to

Dr Martin H Voss;

vossm@mskcc.org

\section{ABSTRACT}

Therapeutic combinations of VEGFR tyrosine kinase inhibitor plus immune checkpoint blockade now represent a standard in the first-line management of patients with advanced renal cell carcinoma. Tumor molecular profiling has shown notable heterogeneity when it comes to activation states of relevant pathways, and it is not clear that concurrent pursuit of two mechanisms of action is needed in all patients. Here, we applied an in silico drug model to simulate combination therapy by integrating previously reported findings from individual monotherapy studies. Clinical data was collected from prospective clinical trials of axitinib, cabozantinib, pembrolizumab and nivolumab. Efficacy of two-drug combination regimens (cabozantinib plus nivolumab, and axitinib plus pembrolizumab) was then modeled assuming independent effects of each partner. Reduction in target lesions, objective response rates (ORR), and progression-free survival (PFS) were projected based on previously reported activity of each agent, randomly pairing efficacy data from two source trials for individual patients and including only the superior effect of each pair in the model. In silico results were then contextualized to register phase III studies of these combinations with similar ORR, PFS, and best tumor response. As increasingly complex therapeutic strategies emerge, computational tools like this could help define benchmarks for trial designs and precision medicine efforts. Summary statement: In silico drug modeling provides meaningful insights into the effects of combination immunotherapy for patients with advanced kidney cancer.

\section{INTRODUCTION}

Combination vascular endothelial growth factor receptor (VEGFR) tyrosine kinase inhibitor (TKI) plus immuno-oncology (IO) therapy constitutes a first-line standard for metastatic renal cell carcinoma (mRCC), with survival benefit over TKI monotherapy. ${ }^{12}$ IO monotherapy similarly achieves less striking efficacy compared with combinations. Transcriptomic and immunogenomic data highlight interpatient tumor heterogeneity and specific activation states of targeted molecular pathways pertinent for individual drug activity. ${ }^{3}$ To evaluate how these drugs combine, we performed in silico modeling ${ }^{4}$ integrating monotherapy clinical data to simulate TKI/IO combinations.

\section{METHODS}

Objective response rates (ORR) and progression-free survival (PFS) data were collected from seven individual mRCC prospective studies conducted in TKI-naïve and immune checkpoint inhibitor naïve patients: monotherapy trials of axitinib, cabozantinib, nivolumab, and pembrolizumab; and, registration studies of axitinib plus pembrolizumab and cabozantinib plus nivolumab. ${ }^{125-9}$ Monotherapy data were digitally measured using Plot Digitizer (V.2.6.9) and a custom Python script was written to extrapolate probability density function from each PFS curve. Each in silico patient was randomly assigned a PFS, response (yes/no), and maximal tumor reduction for each of the two drugs using published monotherapy data. Combination therapy was modeled under the assumption of independent treatment effects, hypothesizing that solely the more active of the two agents was determining the extent of benefit. Thus, for ORR, PFS and best response, each in silico patient was assigned the best values of the two individual therapies. Each trial was run 1000 times, unless otherwise stated, with a median PFS resultant from each simulation. Empiric 95\% CI estimates were calculated from each run.

\section{RESULTS}

Monotherapy source data including ORR, PFS and overall tumor volume change were digitally measured from available clinical trials (see online supplemental figure 1 for measurements). To first test the model, we calculated single agent ORR and a PFS distribution for each of the four agents (online supplemental figure 2). Next, using this in 
Progression-Free Survival Estimates

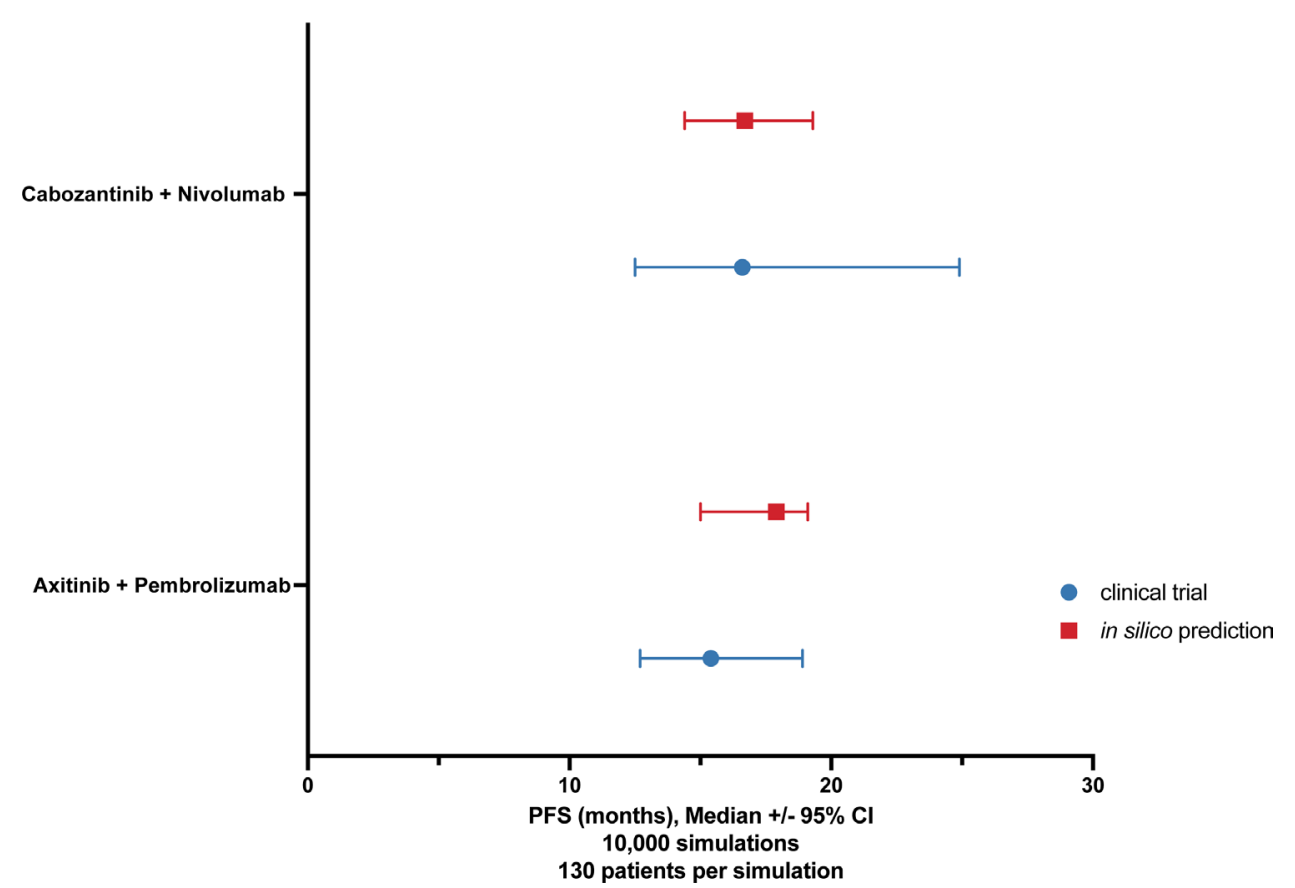

Figure 1 Forest plot of progression-free survival (PFS) of combination TKI/IO therapy. In silico combination (red) and published registration data (blue) reported, with $95 \% \mathrm{Cl}$ estimates. 10 , immuno-oncology; TKI, tyrosine kinase inhibitor.

silico monotherapy data, we modeled outcomes of the two combination therapies. The in silico median PFS for axitinib/pembrolizumab and cabozantinib/nivolumab were 17.9 months (95\% CI 15.0 to 19.1 ) and 16.7 months (95\% CI 14.4 to 19.3 ), respectively. Figure 1 contextualizes these findings with PFS data from the published registration studies. Best radiographic responses in target lesions are summarized in figure 2 (together with data from published registration trials, online supplemental figure 3). In silico ORR for axitinib/pembrolizumab was $75.6 \%$ (95\% CI 70.6 to 80.5 ) with $17.1 \%$ of patients achieving $100 \%$ target regression (95\% CI 12.9 to 21.3).

A

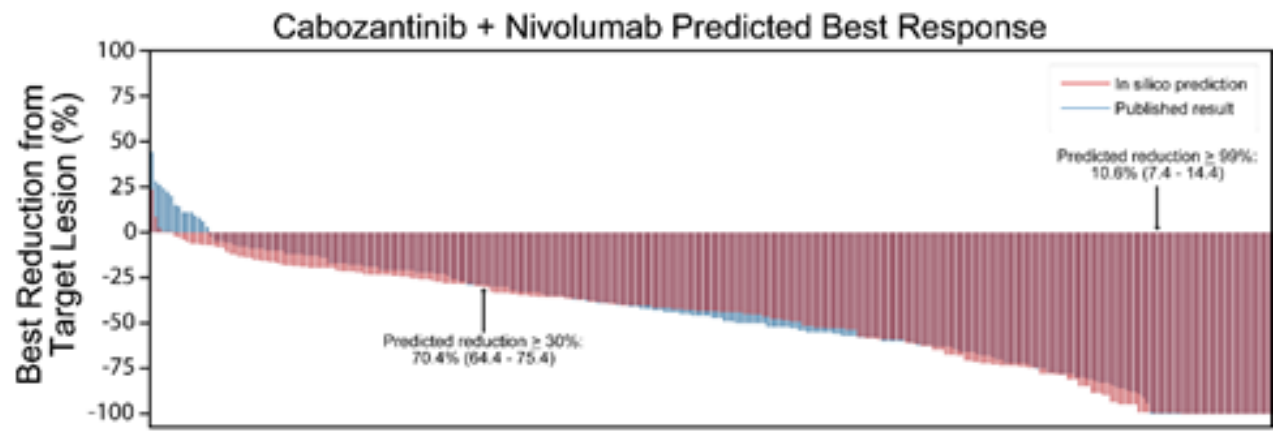

B

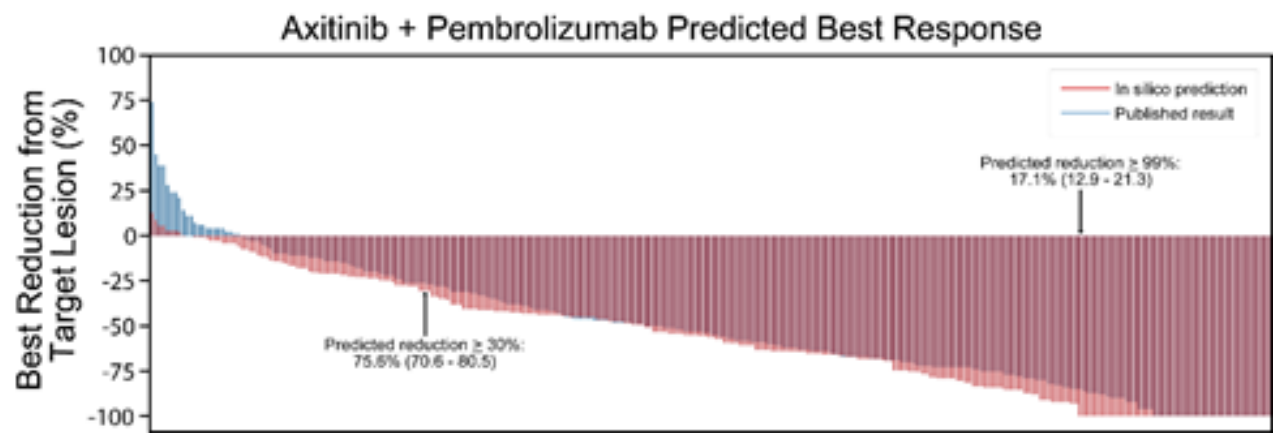

Figure 2 (A-B) Waterfall plot of best response of combination therapy. In silico combination (red) and published registration data (blue) are superimposed for combination cabozantinib plus nivolumab and axitinib plus pembrolizumab. 
For in silico cabozantinib/nivolumab, the model estimated an ORR of $70.4 \%$ (95\% CI $64.4 \%$ to $75.4 \%$ ) with $10.6 \%$ achieving $100 \%$ target regression (95\% CI 7.4 to $14.4)$.

\section{DISCUSSION}

As more complex therapeutic strategies emerge for patients with mRCC (eg, triplicate therapy), clinical tools which estimate efficacy thresholds are needed for clinical trial design and for benchmarking combination strategies. TKI/IO combinations have revolutionized outcomes for patients with mRCC, and superiority has been proposed to stem from the immunomodulatory effects of TKI which may promote IO activity. To estimate the effects of combining agents, other computational models have previously been applied to assess the contribution of individual drug activity with IO therapy. ${ }^{10}$ Here, we performed in silico modeling of TKI/IO combinations by integrating monotherapy clinical trial data. Through random pairing of response and 'best of two' read-outs (which ignore additive treatment effects), our model produced ORR and PFS data comparable to TKI/ IO registration data. Undoubtedly, differences in study populations, dosing levels, and our inability to model how treatment-related toxicities affect drug exposure and treatment duration limit the accuracy of these projections. In addition, as the model relies on selection of the 'best-of-two' treatment response, it is limited in simulating progressive disease as it underestimates response in patients with tumors harboring cross-resistance to both TKI and IO therapies. Nevertheless, these findings highlight how some patients may derive therapeutic benefit from early concurrent exposure to independent mechanisms of action with these agents. As the field looks forward to building on these doublet backbone therapeutic regimens, incorporating in silico modeling may allow for an enhanced appreciation of how these agents combine and may allow for early identification of combinations which harbor negative interacting effects when clinical data deviate from modeled simulations. Lastly, these results call for further translational efforts which integrate biomarkers to adaptively match therapies with tumor phenotypes dynamically.

\section{The following software/libraries were used}

- NumPy: Harris, C.R., Millman, K.J., van der Walt, S.J. et al. Array programming with NumPy. Nature 585, 357-362 (2020). DOI: 0.1038/s41586-020-2649-2.

- lifelines: Cameron Davidson-Pilon, Jonas Kalderstam, Noah Jacobson, Sean Reed, Ben Kuhn, Paul Zivich, ... Dave Golland. (2021, March 3). CamDavidsonPilon/ lifelines: 0.25.10 (Version v0.25.10). Zenodo. http:// doi.org/10.5281/zenodo.4579431

- Python V.3.7.3

- pandas: Jeff Reback, Wes McKinney, jbrockmendel, Joris Van den Bossche, Tom Augspurger, Phillip Cloud, .. Mortada Mehyar. (2020, March 18). pandas-dev/ pandas: Pandas V.1.0.3. Zenodo. http://doi.org/10. 5281/zenodo.3715232

- Plot Digitzer (V.2.6.9). Source code for program is available: http://plotdigitizer.sourceforge.net/

Twitter Dennis J Hsu @DennisJHsu

Contributors Authors confirm contribution to the paper as follows: study contribution and design: RRK, DH, MHV. Data collection and analysis; interpretation of results; manuscript preparation: all authors. All authors reviewed and approved the final version of the manuscript.

Funding The authors have not declared a specific grant for this research from any funding agency in the public, commercial or not-for-profit sectors. Ritesh R. Kotecha, MD is supported (in part) by the Academy of Kidney Cancer Investigators of the CDMRP/DOD (KC200127). Dennis J. Hsu, MD, is a Damon Runyon PhysicianScientist supported (in part) by the Damon Runyon Cancer Research Foundation (PST-31-20).

Competing interests RRK reports advisory board consultation for Eisai and reports receiving institutional research funding from Pfizer and Takeda. MHV reports receiving commercial research support from Bristol-Myers Squibb, Pfizer and Genentech/Roche; honoraria from Novartis and Bristol-Myers Squibb; travel/ accommodation from Astra Zeneca, Eisai, Novartis and Takeda; consultant/ advisory board member for Aveo, Calithera Biosciences, Corvus Pharmaceuticals, Exelixis, Eisai, Merck, Onquality Pharmaceuticals, Novartis and Pfizer. C-HL reports consulting fees from Amgen, Bristol Myers Squibb, Exelixis, Pfizer, Eisai, Merck and EMD Serono; honoraria from AiCME, Intellisphere, and Research to Practice; institutional research support from Bristol Myers Squibb, Calithera, Eisai, Eli Lilly, Exelixis, Merck, and Pfizer. The remaining authors have no competing interests to declare.

Patient consent for publication Not applicable.

Ethics approval This study does not involve human participants.

Provenance and peer review Not commissioned; externally peer reviewed.

Supplemental material This content has been supplied by the author(s). It has not been vetted by BMJ Publishing Group Limited (BMJ) and may not have been peer-reviewed. Any opinions or recommendations discussed are solely those of the author(s) and are not endorsed by BMJ. BMJ disclaims all liability and responsibility arising from any reliance placed on the content. Where the content includes any translated material, BMJ does not warrant the accuracy and reliability of the translations (including but not limited to local regulations, clinical guidelines, terminology, drug names and drug dosages), and is not responsible for any error and/or omissions arising from translation and adaptation or otherwise.

Open access This is an open access article distributed in accordance with the Creative Commons Attribution Non Commercial (CC BY-NC 4.0) license, which permits others to distribute, remix, adapt, build upon this work non-commercially, and license their derivative works on different terms, provided the original work is properly cited, appropriate credit is given, any changes made indicated, and the use is non-commercial. See http://creativecommons.org/licenses/by-nc/4.0/.

\section{ORCID iDs}

Ritesh R Kotecha http://orcid.org/0000-0002-0223-3479

Dennis J Hsu http://orcid.org/0000-0003-4200-1098

Martin H Voss http://orcid.org/0000-0003-0551-5807

\section{REFERENCES}

1 Rini BI, Plimack ER, Stus V, et al. Pembrolizumab plus axitinib versus sunitinib for advanced renal-cell carcinoma. N Engl J Med Overseas Ed 2019;380:1116-27.

2 Choueiri TK, Powles T, Burotto M, et al. Nivolumab plus cabozantinib versus sunitinib for advanced renal-cell carcinoma. $N$ Engl J Med Overseas Ed 2021;384:829-41.

3 Motzer RJ, Banchereau R, Hamidi H, et al. Molecular subsets in renal cancer determine outcome to checkpoint and angiogenesis blockade. Cancer Cell 2020;38:803-17.

4 Palmer AC, Sorger PK. Combination cancer therapy can confer benefit via patient-to-patient variability without drug additivity or synergy. Cell 2017;171:1678-91.

5 Hutson TE, Al-Shukri S, Stus VP, et al. Axitinib versus sorafenib in first-line metastatic renal cell carcinoma: overall survival from a randomized phase III trial. Clin Genitourin Cancer 2017;15:72-6. 
6 McDermott DF, Lee J-L, Bjarnason GA, et al. Open-label, single-arm phase II study of pembrolizumab monotherapy as first-line therapy in patients with advanced clear cell renal cell carcinoma. J Clin Oncol 2021;39:1020-8.

7 Choueiri TK, Hessel C, Halabi S, et al. Cabozantinib versus sunitinib as initial therapy for metastatic renal cell carcinoma of intermediate or poor risk (alliance A031203 CABOSUN randomised trial): progression-free survival by independent review and overall survival update. Eur J Cancer 2018;94:115-25.
8 Atkins MB, Jegede O, Haas NB, et al. Phase II study of nivolumab and salvage nivolumab + ipilimumab in treatment-naïve patients (PTS) with advanced renal cell carcinoma (RCC) (HCRN GU16-260). J Clin Oncol 2020;38:5006.

9 Rixe O, Bukowski RM, Michaelson MD, et al. Axitinib treatment in patients with cytokine-refractory metastatic renal-cell cancer: a phase II study. Lancet Oncol 2007:8:975-84.

10 Schmidt EV, Chisamore MJ, Chaney MF, et al. Assessment of clinical activity of PD-1 checkpoint inhibitor combination therapies reported in clinical trials. JAMA Netw Open 2020;3:e1920833 